\title{
Pien Tze Huang inhibits liver metastasis by targeting TGF- $\beta$ signaling in an orthotopic model of colorectal cancer
}

\author{
WEI LIN ${ }^{1,2}$, QUNCHUAN ZHUANG ${ }^{1,2}$, LIANGPU ZHENG ${ }^{1,2}$, ZHIYUN CAO $^{1,2}$, ALING SHEN $^{1}$, \\ QIONGYU LI ${ }^{1}$, CAIXUAN FU ${ }^{1}$, JIANYU FENG ${ }^{1}$ and JUN PENG ${ }^{1,2}$ \\ ${ }^{1}$ Academy of Integrative Medicine Biomedical Research Center, Fujian University of Traditional Chinese Medicine; \\ ${ }^{2}$ Fujian Key Laboratory of Integrative Medicine on Geriatrics, Fujian University of \\ Traditional Chinese Medicine, Fuzhou, Fujian 350122, P.R. China
}

Received October 23, 2014; Accepted January 19, 2015

DOI: $10.3892 /$ or.2015.3784

\begin{abstract}
Metastasis is the leading cause of cancer-related mortality in almost all types of cancers, including colorectal cancer (CRC). Epithelial-mesenchymal transition (EMT) is a critical process during the metastatic cascade. This process may be a potential target for the diagnosis and treatment of CRC.Pien Tze Huang (PZH), a well-known traditional Chinese formula, has been demonstrated to be clinically effective in treating various types of human malignancies, including CRC. Our published data suggest that $\mathrm{PZH}$ can induce apoptosis, as well as inhibit cell proliferation and tumor angiogenesis, thus suppressing CRC growth in vitro and in vivo. We evaluated the therapeutic efficacy of $\mathrm{PZH}$ against $\mathrm{CRC}$ metastasis using a CRC liver metastasis mouse model to further explore the mechanisms underlying the antitumor action of PZH. MTT, migration, and Matrigel invasion assays were used to assess the effect of PZH on cell viability, migration and invasion. We then established an orthotopic liver metastasis model of colon cancer using microsurgical techniques. Mice were intragastrically administered $234 \mathrm{mg} / \mathrm{kg} /$ day dose of either PZH or saline for 14 days. The body and tumor weights of the mice were measured after they were sacrificed. Moreover, we examined the effect of PZH inhibition on liver metastasis. Finally, EMT-related proteins and the TGF- $\beta$ signaling pathway were assessed using immunohistochemical staining (IHS). The present data revealed that $\mathrm{PZH}$ significantly inhibited the migration and invasion of CT-26 cells in a dose-dependent
\end{abstract}

Correspondence to: Dr Jun Peng, Academy of Integrative Medicine Biomedical Research Center, Fujian University of Traditional Chinese Medicine, 1 QiuYangXi Road, Minhou Shangjie, Fuzhou, Fujian 350122, P.R. China

E-mail: pjunlab@hotmail.com

Abbreviations: CRC, colorectal cancer; PZH, Pien Tze Huang; EMT, epithelial-mesenchymal transition; TCM, traditional Chinese medicine; TGF- $\beta$, transforming growth factor- $\beta$;

Key words: $\mathrm{PZH}$, colorectal cancer, metastasis, TGF- $\beta$ pathway, EMT manner, which affirmed the inhibitory effect of PZH on CRC cell metastasis. No significant change was observed between the in vivo primary tumor growth and body weight. However, the control group had five cases of liver metastasis $(5 / 6)$, whereas one case was found in the PZH group (1/6). Thus, PZH exhibited therapeutic efficacy against CRC metastasis without apparent toxicity. The inhibitory effect of PZH on EMT resulted in an increase in E-cadherin expression, as well as a decrease in $\mathrm{N}$-cadherin expression. In addition, $\mathrm{PZH}$ significantly inhibited TGF- $\beta$, as well as the phosphorylation of Smad2/3 and Smad4 in the tumor tissues, indicating its suppressive action on TGF- $\beta$ signaling. These molecular effects ultimately resulted in the inhibition of cancer cell EMT and tumor metastasis.

\section{Introduction}

Metastasis is the leading cause of cancer-related mortality in almost all types of cancers, including colorectal cancer (CRC). Although surgery, chemotherapy and radiotherapy have long been the standards of CRC treatment, the long-term survival and prognosis of patients remain quite poor due to tumor recurrence and metastasis $(1,2)$. Over $50 \%$ of patients with CRC present with liver metastases or develop liver metastases (3). Thus, CRC remains a major global public health issue.

Metastasis is a complex, multistep and dynamic biological process, with epithelial-mesenchymal transition (EMT) being a critical step during the cascade (4). EMT refers to the morphological and molecular changes that occur when epithelial cells lose their characteristics, gain mesenchymal properties and become motile, which makes EMT a key event in tumor invasion and metastasis (5-7). EMT could thus be a potential target for CRC therapy.

Some complementary and alternative medical systems, such as traditional Chinese medicine (TCM), have various approaches to cancer treatment. TCM has the advantage of reducing cancer therapy-induced toxicity (8). The modified classic formula has been shown to further minimize the side effects of surgery, radiation and chemotherapy. This formula also increases immunity and improves survival (9-11). Therefore, novel therapeutic strategies are essential to improve the clinical management of patients with CRC. 
Pien Tze Huang $(\mathrm{PZH})$ was first prescribed by a royal physician in the Ming Dynasty. PZH is a well-known traditional Chinese formulation that is popularly known in Southeast Asia. The main ingredients of PZH include Moschus, Calculus Bovis, Snake Gall and Radix Notoginseng. These products ensure that PZH has heat clearing, detoxification, blood circulation promotion, blood stasis reduction and swelling reduction effects (12). Modern pharmacological studies have proposed that $\mathrm{PZH}$ not only exhibits therapeutic effects on hepatocellular carcinoma and CRC in clinical trials $(13,14)$, but also inhibits the growth of human colon carcinoma cells by activating mitochondrion-dependent apoptosis (15). Moreover, PZH reportedly suppressed the cell proliferation and tumor angiogenesis of CRC carcinoma cells in vitro and in vivo $(16,17)$. To elucidate further the antitumor mechanism of action of $\mathrm{PZH}$, we evaluated its efficacy against tumor invasiveness in vivo and in vitro and investigated its underlying molecular mechanisms.

\section{Materials and methods}

Materials and methods. Dulbecco's modified Eagle's medium (DMEM), fetal bovine serum (FBS), penicillin-streptomycin, and trypsin-EDTA were purchased from Invitrogen (Carlsbad, CA, USA). E-cadherin, N-cadherin, TGF- $\beta$, Smad2/3, P-Smad2/3, Smad4 and horseradish peroxidase (HRP)-conjugated secondary antibodies were obtained from Cell Signaling Technology (Beverly, MA, USA). All the other chemicals used, unless otherwise indicated, were obtained from Sigma Chemicals (St. Louis, MO, USA).

Preparation of $\mathrm{PZH}$. PZH was obtained from and authenticated by the sole manufacturer Zhangzhou Pien Tze Huang Pharmaceutical Co., Ltd. (Zhangzhou, China; Chinese FDA, approval no. Z35020242). PZH stock solution was prepared by dissolving $\mathrm{PZH}$ powder in phosphate-buffered saline (PBS) to a concentration of 20 and $234 \mathrm{mg} / \mathrm{ml}$ before use. The PZH working solutions were prepared by diluting the stock solution in either culture medium or saline.

Cell culture. Murine colon carcinoma CT-26 cells were obtained from the Shanghai Cell Bank of the Chinese Academy of Sciences. The cells were grown in DMEM containing 10\% (v/v) FBS, $100 \mathrm{U} / \mathrm{ml}$ of penicillin and $100 \mu \mathrm{g} / \mathrm{ml}$ of streptomycin in a $37^{\circ} \mathrm{C}$ humidified incubator under $5 \% \mathrm{CO}_{2}$. The cells were subcultured at 80 to $90 \%$ confluency.

Cell viability evaluation. Cell viability was assessed through an MTT colorimetric assay. CT-26 cells were seeded into 96-well plates at a density of $1 \times 10^{4}$ cells/well in $0.1 \mathrm{ml}$ of medium. The cells were treated with various concentrations of $\mathrm{PZH}$ for different periods. At the end of the treatment, $100 \mu \mathrm{l}$ of MTT $(0.5 \mathrm{mg} / \mathrm{ml}$ in PBS) was added to each well. The samples were then incubated for an additional $4 \mathrm{~h}$ at $37^{\circ} \mathrm{C}$. The purple-blue MTT formazan precipitate was dissolved in $100 \mu \mathrm{l}$ of DMSO. Absorbance was measured at $570 \mathrm{~nm}$ using an ELISA reader (Model ELx800; BioTek, Winooski, VT, USA).

Migration assay. The migration assay was performed using Transwell cell culture chambers. Conditioned medium (500 $\mu \mathrm{l}$ of media with $10 \%$ FBS) was added to the lower compartment of the chamber. CT-26 cells $\left(5 \times 10^{5}\right)$ in $1 \%$ FBS-containing media were added to the upper compartment of the chamber. After $24 \mathrm{~h}$ of incubation, the top side of the insert membrane was scrubbed with a cotton swab, whereas the bottom side was fixed with ice-cold methanol, stained with $0.01 \%$ crystal violet and scored under a fluorescence microscope (Olympus IX51 with DP70; Olympus, Center Valley, PA, USA).

Matrigel invasion assay. The invasion assay was performed using Transwell cell culture chambers (Corning Costar No. 3422; Corning, Tewksbury, MA, USA) in accordance with the manufacturer's instructions but with specific modifications. Briefly, the upper surface of polyvinylpyrrolidone-free polycarbonate filters $(8.0-\mathrm{mm}$ pore size; Nuclepore Corp., Pleasanton, CA, USA) was pre-coated with $15 \mu 1$ of ice-cold Matrigel (BD Biosciences, Bedford, MA, USA) for $60 \mathrm{~min}$ at room temperature. Conditioned medium (500 $\mu \mathrm{l}$ of medium with $10 \%$ FBS) was added to the lower compartment of the chamber. CT-26 cells $\left(5 \times 10^{5}\right)$ in $1 \%$ FBS-containing media were added to the upper compartment of the chamber. After $24 \mathrm{~h}$ of incubation, the top side of the membrane insert was scrubbed free of cells using a cotton swab, whereas the bottom side was fixed with ice-cold methanol, stained with $0.01 \%$ crystal violet and quantified.

Animals. Male BALB/c athymic (nude) mice and BALB/c mice (with an initial body weight of 20 to $22 \mathrm{~g}$ ) were obtained from Shanghai SLAC Laboratory Animal Co., Ltd. (Shanghai, China) and housed under pathogen-free conditions with controlled temperature $\left(22^{\circ} \mathrm{C}\right)$, humidity and a 12 -h light/ dark cycle. Food and water were given ad libitum throughout the experiment. All the animal treatments were performed in strict accordance with international ethical guidelines and the National Institutes of Health Guide Concerning the Care and the Use of Laboratory Animals. The experiments were approved by the Institutional Animal Care and Use Committee of Fujian University of Traditional Chinese Medicine.

CRC liver metastasis animal model. CT-26 cells were grown in culture and then detached through trypsinization. Thereafter, the cells were washed and resuspended in serum-free DMEM. Then, $1.5 \times 10^{6}$ of cells mixed with Matrigel (1:1) were subcutaneously injected into the right flank area of athymic nude mice, the growth of which was monitored regularly. After 16 days of xenograft implantation, tumors were selected for implantation to other nude mice using a 20-inoculating needle at an average size of $1 \mathrm{~cm}^{3}$. The tumor tissues of 4-6 generations were chosen for orthotopic transplantation into the cecum surface. Mice were anesthetized with an i.p. injection of Nembutal (pentobarbital; Abbott Laboratories, Abbott Park, IL, USA) at a dose of $70 \mathrm{mg} / \mathrm{kg}$. After shaving, a small incision was made through their skin over the cecum under a dissecting microscope and by using microsurgical techniques. The cecum was exteriorized through a small midline laparotomy, and a piece of tumor tissue that was derived from CT-26 cells was sutured to the cecal surface with a single Maxon 11/0 suture, which left the tumor tissue buried in a 'pouch' that consisted of a double cecal wall on each side. After implantation, the abdominal wall was closed in two layers with Dexon 5/0. Food and water 


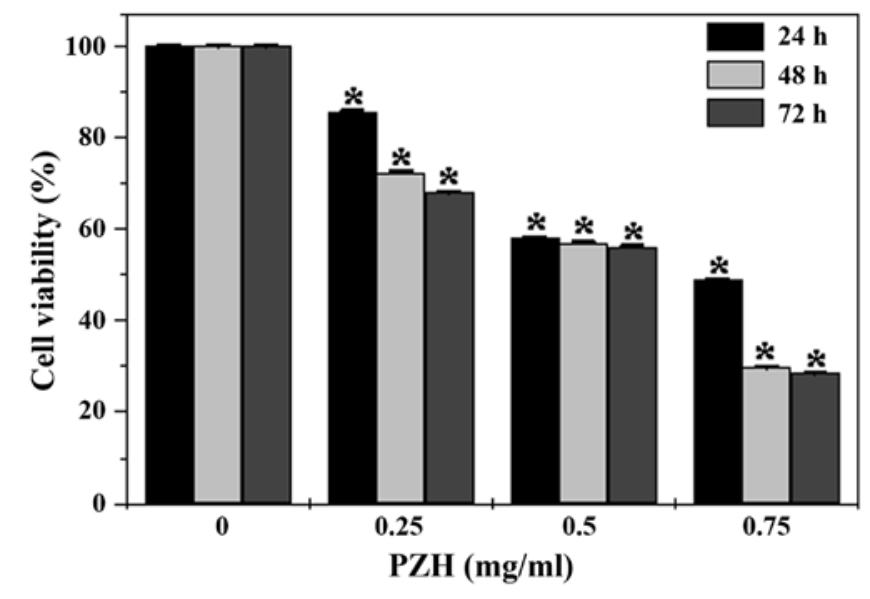

Figure 1. Effect of PZH on the viability of CT-26 cells. The cells were treated with various concentrations of $\mathrm{PZH}$ for the indicated time-periods. Cell viability was determined using an MTT assay. The data were normalized to the viability of the control cells ( $100 \%$, treated with $0.5 \%$ DMSO vehicle). Data are presented as the mean \pm standard deviation from 3 independent experiments. ${ }^{*} \mathrm{P}<0.05$ vs. the control cells. PZH, Pien Tze Huang.

were given ad libitum. A few days after the cecal implantation of the colon tumor, the groups of mice were randomly assigned to receive one of two treatments ( $n=6 /$ group). The groups of mice were intragastrically administered with a $234 \mathrm{mg} / \mathrm{kg} /$ day dose of either PZH or saline daily. The animals were sacrificed 14 days after tumor implantation. The tumor tissues were removed and weighed. A portion of each tumor was fixed in $10 \%$ buffered formalin. The remaining tissues were snap-frozen in liquid nitrogen and stored at $-80^{\circ} \mathrm{C}$. We recorded all the macroscopic tumor deposits and abnormalities in the liver. The number of liver metastases was calculated. All neoplasms were identified through H\&E staining.

Histological examination by $H \& E$ staining. The metastasized liver tissues were fixed with $10 \%$ buffered formalin for $24 \mathrm{~h}$. Samples were then paraffin-embedded, sectioned and stained with H\&E. Histopathological changes were observed under a light microscope.

Immunohistochemistry. Immunohistochemical staining for E-cadherin, N-cadherin, TGF- $\beta, \operatorname{Smad} 2 / 3, \mathrm{P}-\mathrm{Smad} 2 / 3$ and Smad4 was performed as follows. Briefly, after being fixed with $10 \%$ formaldehyde for $12 \mathrm{~h}$, tumor samples were processed for paraffin-embedded tumor slides. The slides were subjected to antigen retrieval, and the endogenous peroxidase activity was quenched with hydrogen peroxide. After blocking nonspecific proteins with normal serum in PBS (0.1\% Tween-20), the slides were incubated with rabbit polyclonal antibodies against E-cadherin, N-cadherin, Smad2/3, P-Smad2/3, Smad4, TGF- $\beta$, ZEB1 and ZEB2 (all in 1:200 dilution). After being washed with PBS, the slides were incubated with a biotinylated secondary antibody followed by conjugated HRP-labeled streptavidin (Dako) and then washed with PBS. The slides were then incubated with diaminobenzidine (DAB, Sigma Chemicals) as the chromogen, followed by counterstaining with diluted Harris' hematoxylin (Sigma Chemicals). After staining, five 5-power fields (400x) were randomly selected for each slide. The average proportion of the positive cells in each field was quantified using the True Color Multi-Functional Cell Image Analysis Management System (Image-Pro Plus; Media Cybernetics, Rockville, MD, USA). To rule out any non-specific staining, PBS was used to replace the primary antibody as a negative control.

Statistical analysis. All data are the means of 3 determinations and were analyzed using SPSS Package for Windows (Version 11.5). Statistical data analysis was performed using Student's t-test and ANOVA. Differences with $\mathrm{P}<0.05$ were considered statistically significant.

\section{Results}

PZH inhibits the viability of CT-26 cells. The viability of the CT-26 cells was examined using an MTT assay to compare the relative number of cells in the PZH-treated monolayers with that in the untreated controls. As shown in Fig. 1, treatment with 0.25 to $0.75 \mathrm{mg} / \mathrm{ml}$ of PZH for 24,48 and $72 \mathrm{~h}$, respectively reduced cell viability by 14.3 to $51.1 \%, 27.4$ to $60.3 \%$, and 32.1 to $71.5 \%$ compared with the untreated control cells $(\mathrm{P}<0.05)$. These data indicate that PZH inhibited CT-26 cell growth and proliferation in a dose- and time-dependent manner.

PZH inhibits migration and invasion of CT-26 cells. To examine the effects of PZH on cell migration, we performed Transwell migration assays with CT-26 cells. As demonstrated in Fig. 2 (upper panels), PZH effectively inhibited cell migration of CT-26 cells in a dose-dependent manner. Furthermore, a Transwell invasion assay was used to determine the invasive activity of the tumor cells across the basement membrane (Fig. 2, lower panels). Overall, the results revealed that PZH significantly decreased the invasive potential of CT-26 cells in a dose-dependent manner.

PZH inhibits tumor liver metastasis in the orthotopic CRC mouse model. The antitumor effect on liver metastasis of $\mathrm{PZH}$ in vivo was determined by examining the tumor weight and the number of liver metastases in the orthotopic CRC mouse model. The adverse effects were evaluated by measuring body weight changes. As shown in Fig. 3A and B, PZH treatment significantly reduced the number of tumor liver metastases as compared with the control $(\mathrm{P}<0.01)$. After $\mathrm{H} \& \mathrm{E}$ staining, histological changes in the liver samples of CRC mice were observed under light microscopy. PZH treatment was observed to have no effect on the changes in the tumor and body weights of the mice (Fig. 3C). Thus, PZH effectively suppressed CRC liver metastasis in vivo, but did not significantly affect CRC growth and caused no apparent signs of toxicity.

PZH inhibits EMT in the orthotopic CRC mouse model. E-cadherin and N-cadherin, which are markers of EMT, not only mediate cell migration and invasion properties, but also contribute to metastasis $(18,19)$. Therefore, we further investigated E-cadherin, $\mathrm{N}$-cadherin, and ZEB1 expression to explore further the anti-metastatic activity of PZH. We performed immunohistochemical staining (IHS) analyses to examine the protein expression of both E-cadherin and $\mathrm{N}$-cadherin 


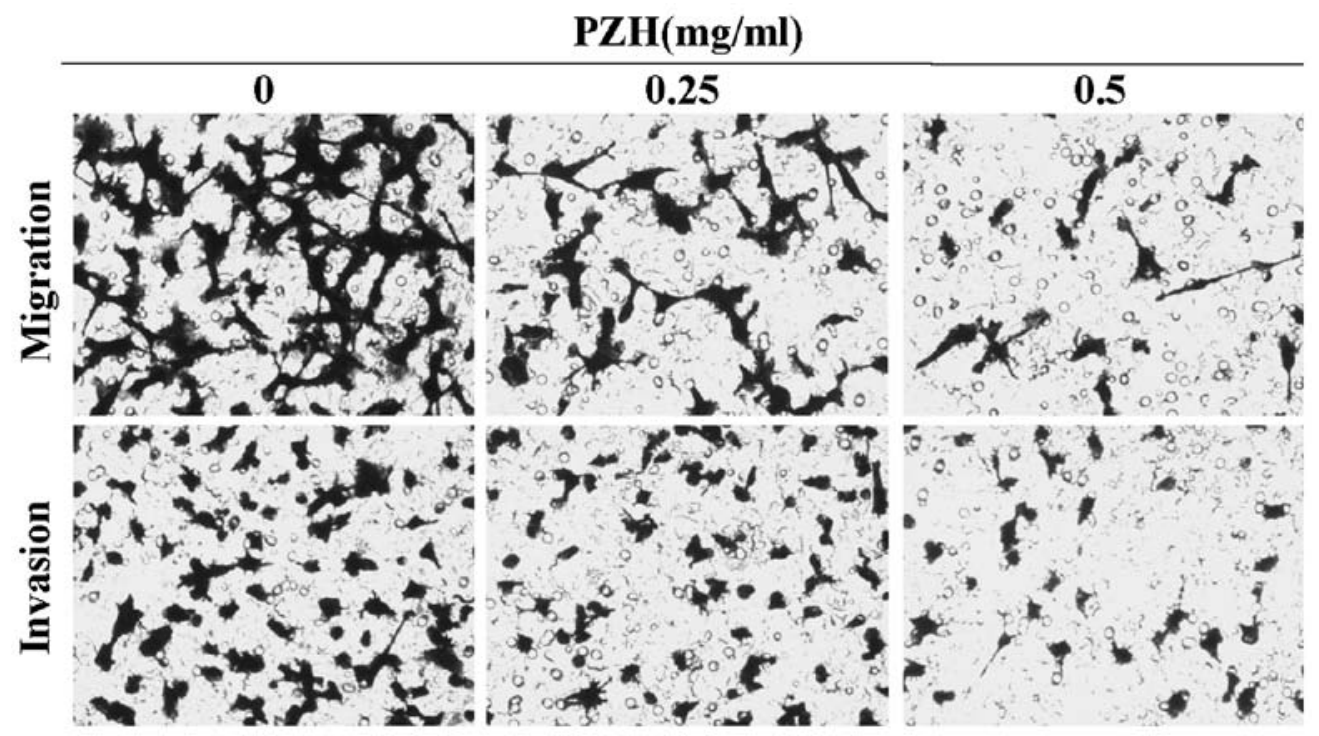

Figure 2. Effect of PZH on CT-26 cell migration and invasion. CT-26 cells were treated with the indicated concentrations of PZH for $6 \mathrm{~h}$. Cell migration was determined using Transwell ${ }^{\circledast}$ cell culture chambers. Cell invasion was determined using Transwell cell culture chambers with membranes coated with Matrigel $^{\mathrm{TM}}$. Cells were fixed and stained with crystal violet, and images were captured at a magnification, $\mathrm{x} 200$. The average number of migrated cells was determined from three randomly selected fields. Data are presented as the mean \pm standard deviation from three independent experiments. PZH, Pien Tze Huang.
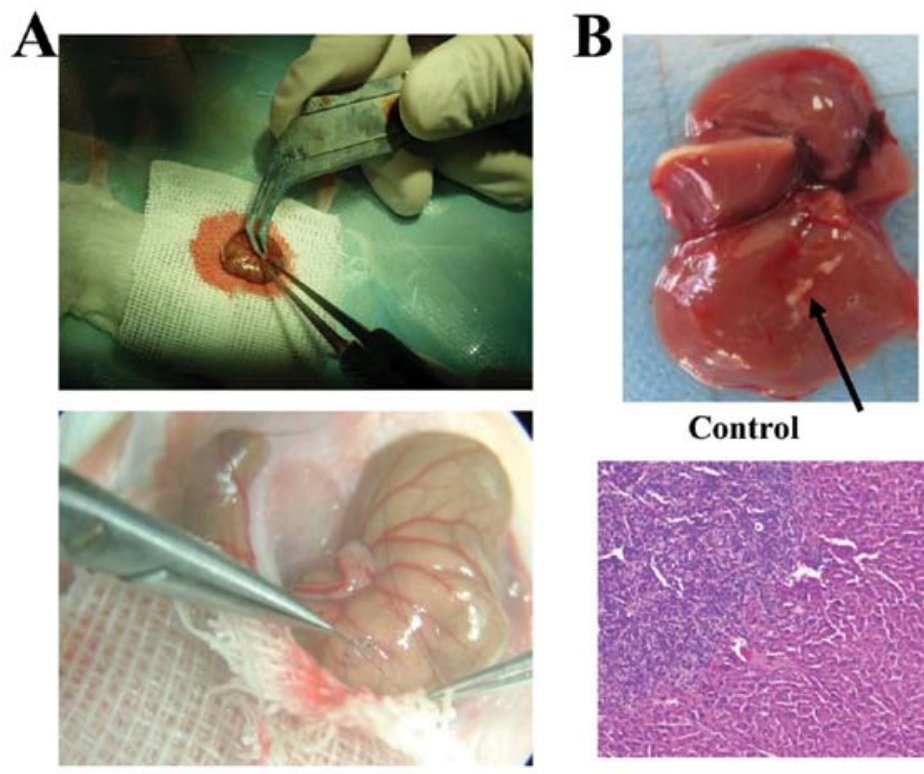

Control

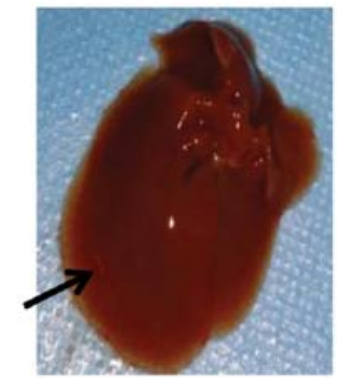

PZH
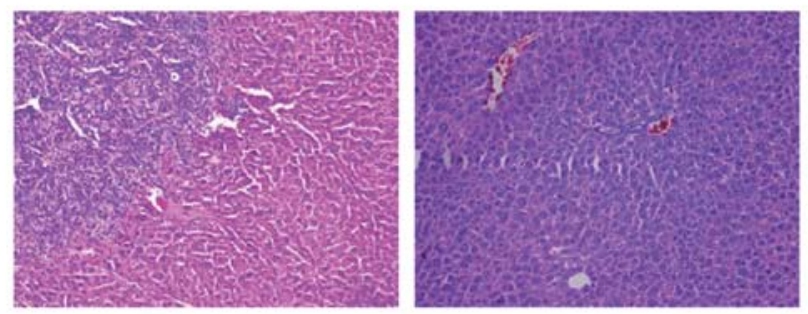

C

\begin{tabular}{|c|c|c|c|c|c|}
\hline \multirow{2}{*}{ Group } & \multirow{2}{*}{$\begin{array}{c}\text { Mice } \\
(\mathrm{n})\end{array}$} & \multicolumn{2}{|c|}{ Local tumor } & \multicolumn{2}{c|}{ Dissemination site [mice (\%)] } \\
\cline { 3 - 6 } & & Take rate [mice (\%)] & Final tumor weight (g) & Hepatic & carcinoma \\
\hline Control & 6 & $6 / 6(100)$ & $0.18 \pm 0.09$ & $5 / 6$ & $5 / 6(83.3)$ \\
\hline PZH & 6 & $6 / 6(100)$ & $0.20 \pm 0.10$ & $1 / 6$ & $1 / 6(16.7)$ \\
\hline
\end{tabular}

Figure 3. Effect of PZH on tumor liver metastasis in the orthotopic CRC mouse model. At the end of the experiment, tumor tissues from the control and the PZH-treated group were measured, and the number of tumor liver metastases was calculated. (A and B) PZH treatment significantly reduced the number of tumor liver metastases (arrows) as compared with the control. Tumor liver metastasis tissues were fixed with $10 \%$ buffered formalin for $24 \mathrm{~h}$. Samples were then paraffin-embedded, sectioned, and stained with H\&E. Histopathological changes were observed under a light microscope. Images are representative images captured at a magnification, $\mathrm{x} 200$. (C) Data are presented as the mean \pm standard deviation from six individual mice in each group. " $\mathrm{P}<0.01$ vs. controls. CRC, colorectal cancer; PZH, Pien Tze Huang.

in the orthotopic CRC mouse model. As shown in Fig. 4, the percentages of E-cadherin-positive cells in the PZH-treated and control mice were $37.50 \pm 5.16$ and $27.17 \pm 5.42 \%(\mathrm{P}<0.01)$, respectively. By contrast, the proportion of cells expressing 

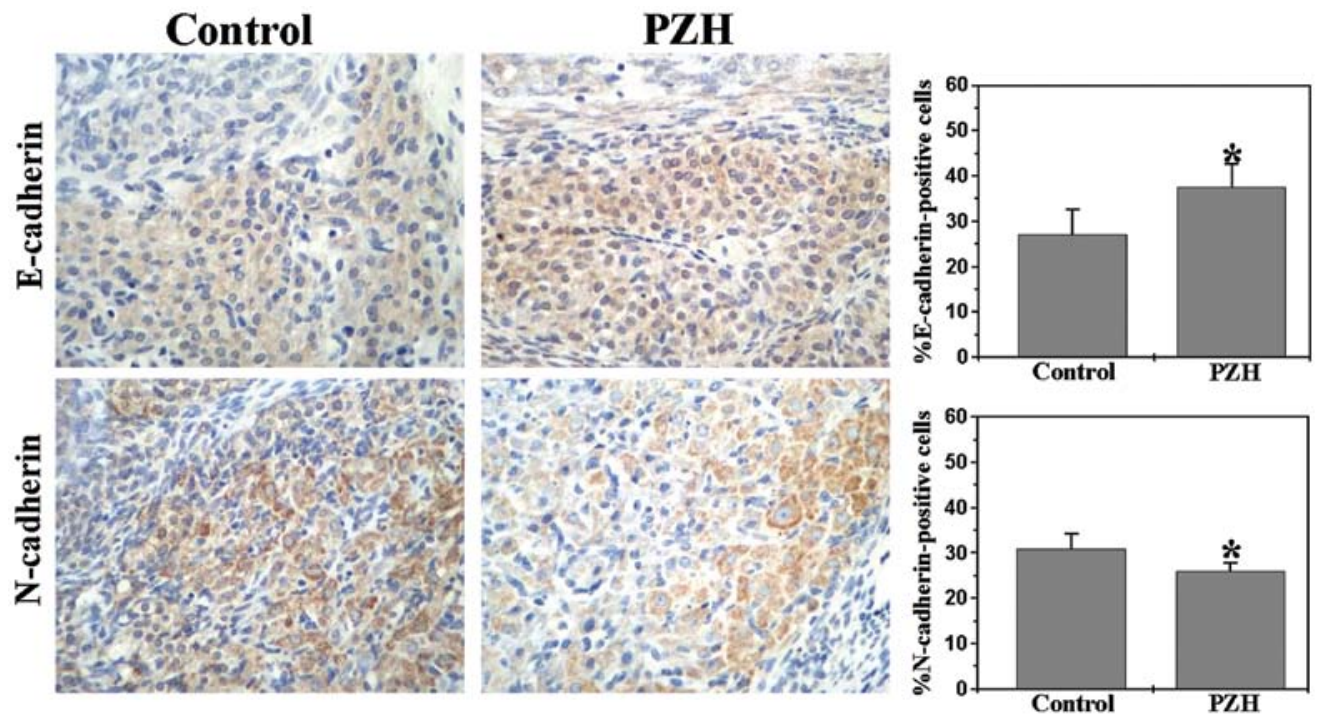

Figure 4. Effect of PZH on EMT in the orthotopic colorectal cancer mouse model. At the end of the experiment, tumor tissues from the control and the $\mathrm{PZH}$-treated group were processed for immunohistochemical staining for E-cadherin and $\mathrm{N}$-cadherin. The photographs are representative images captured at a x400 magnification. Quantification of the immunohistochemical assay is represented as the percentage of the positively stained cells. Data are presented as the mean \pm standard deviation from six individual mice in each group. ${ }^{*} \mathrm{P}<0.01$ vs. controls. PZH, Pien Tze Huang.
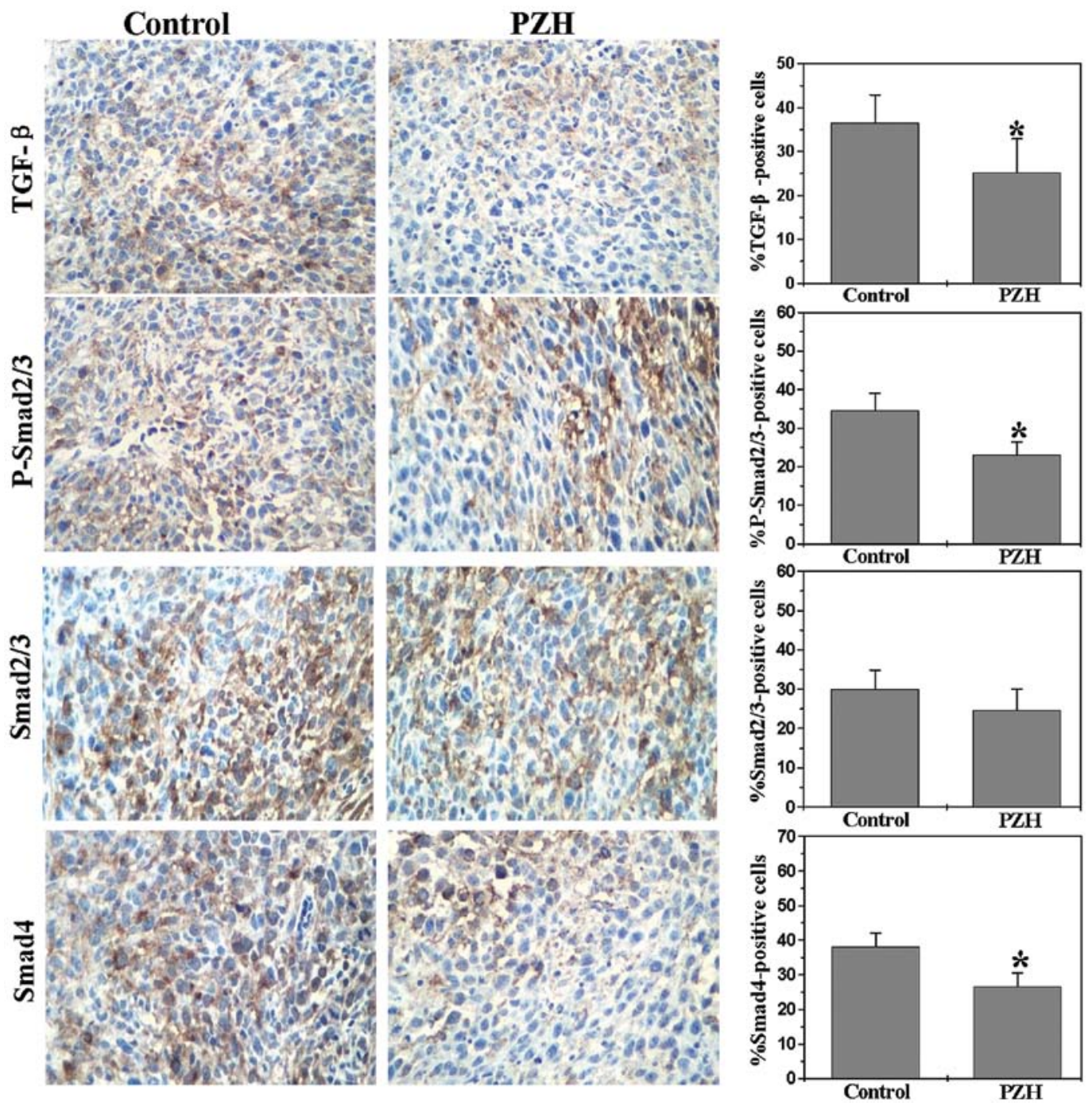

Figure 5. Effect of PZH on the TGF- $\beta /$ Smad pathway in the orthotopic colorectal cancer mouse model. Tumor tissues from the control and the PZH-treated group were also processed for immunohistochemical staining for TGF- $\beta$, Smad2/Smad3, P-Smad2/Smad3 and Smad4. The photographs are representative images captured at a x400 magnification. Quantification of the immunohistochemical assay is represented as the percentage of positively stained cells. Data are presented as the mean \pm standard deviation from six individual mice in each group. " $\mathrm{P}<0.01$ vs. controls. PZH, Pien Tze Huang. 
$\mathrm{N}$-cadherin was $26.01 \pm 1.78 \%$ in the PZH-treated mice and $30.83 \pm 3.43 \%$ in the controls. These data collectively suggest that the antitumor liver metastasis function of PZH may be attributed to the inhibition of EMT of CRC cells.

PZH inhibits activation of the TGF- $\beta$ pathway in the orthotopic CRC mouse model. Numerous pathways have been implicated in colorectal carcinogenesis. Among these pathways is the TGF- $\beta$ /Smad signaling pathway, which is responsible for TGF- $\beta$-mediated cell growth inhibition and apoptosis. TGF- $\beta$ induces angiogenesis, inflammation and EMT, thus providing a beneficial environment for tumor progression and metastasis (20). Therefore, we assessed the effect of PZH on the expression of key mediators of the TGF- $\beta /$ Smad pathway using IHS analyses. As shown in Fig. 5, PZH treatment significantly reduced the protein expression of TGF- $\beta$, P-Smad $2 /$ Smad3, Smad2/Smad3, and Smad4 in the tumor tissues. The percentages of TGF- $\beta$-, P-Smad2/Smad3-, Smad2/Smad3and Smad4-positive cells in the control group were 36.5 \pm 6.41 , $34.5 \pm 4.55,30.0 \pm 4.63$ and $38.17 \pm 3.68 \%$, respectively. By contrast, the percentages of TGF- $\beta-$, P-Smad2/Smad3-, Smad2/Smad3- and Smad4-positive cells in the tumor tissue of PZH-treated mice were $25.2 \pm 7.69,23.0 \pm 3.40,24.67 \pm 5.32$ and $26.67 \pm 3.82 \%$, respectively $(\mathrm{P}<0.01)$. These data suggest that the in vitro inhibitory effect of $\mathrm{PZH}$ on tumor metastasis could be mediated by the suppression of the TGF- $\beta / \mathrm{Smad}$ pathway.

\section{Discussion}

The occurrence of metastases due to tumor progression causes the vast majority of cancer-related deaths. Metastatic progression is a multi-step process that includes the detachment of cancer cells from the primary tumor mass, migration and invasion, thus enabling the re-establishment of malignant cells at distant sites. TGF- $\beta$ is a multifunctional cytokine and is a potent inducer of EMT $(21,22)$. Moreover, TGF- $\beta$ can serve as a tumor suppressor by inhibiting cell proliferation in the early stages of tumor development while promoting metastasis in various cancer models $(23,24)$. CRC is associated with deregulated levels of TGF- $\beta$, which predicts poor prognosis and limited recurrence-free survival. A growing number of in vivo studies have recently shown that the inhibition of TGF- $\beta$ signaling and transcription reduces the metastatic and/or invasive properties of various experimental cancers, including CRC, presumably by preventing the induction of migration and invasion in cancer cells (25-27).

The function of the TGF- $\beta$ signaling pathway depends on the binding of ligands to cell membrane receptors, activating cytoplasm mediators into the nucleus, and regulating expression of their target gene. The Smad pathway is a major transducer of TGF- $\beta$ signaling (28). Smad 2 and Smad3, which are known as receptor-regulated Smads, are both phosphorylated by the type 1 TGF- $\beta$ receptor and form complexes with Smad4 (29). These complexes accumulate in the nucleus of the cell and regulate the transcription of target genes, thus serving critical functions in controlling cell proliferation, differentiation, apoptosis and cell migration.

E-cadherin and N-cadherin are target genes of the TGF- $\beta$ signaling pathway. These genes are important for control- ling cell migration. The malignancy of carcinoma cells is characterized by the loss of both cell-to-cell adhesion and cellular differentiation, which have been repeatedly reported to correlate with E-cadherin downregulation and $\mathrm{N}$-cadherin upregulation $(30,31)$. Dysfunction or loss of E-cadherin and increase in $\mathrm{N}$-cadherin could be attributed to somatic mutations in some tumor types, which include CRC and are associated with the development of invasive carcinoma, metastatic dissemination and poor clinical prognosis. Therefore, the deregulation of E-cadherin and the upregulation of N-cadherin expression may contribute to tumorigenesis.

In conclusion, the present study is the first to demonstrate that PZH inhibits the invasiveness of CRC by suppressing the TGF- $\beta /$ Smad pathway, by promoting the expression of E-cadherin and by suppressing the expression of $\mathrm{N}$-cadherin. Our findings suggest that PZH may be a potential novel therapeutic agent for the treatment of cancers owing to its inhibition of CRC metastasis.

\section{Acknowledgements}

We acknowledge- Projects 81373819 and 81202790 supported by the National Natural Science Foundation of China and Project 2014J01359 by the Natural Science Foundation of Fujian.

\section{References}

1. Jemal A, Siegel R, Xu J and Ward E: Cancer statistics, 2010. CA Cancer J Clin 60: 277-300, 2010.

2. Gansler T, Ganz PA, Grant M, et al: Sixty years of CA: a cancer journal for clinicians. CA Cancer J Clin 60: 345-350, 2010.

3. Jemal A, Thomas A, Murray T and Thun M: Cancer statistics. CA Cancer J Clin 52: 23-47, 2002.

4. Klymkowsky MW and Savagner P: Epithelial-mesenchymal transition: a cancer researcher's conceptual friend and foe. Am J Pathol 174: 1588-1593, 2009.

5. Moustakas A and Heldin CH: Signaling networks guiding epithelial-mesenchymal transitions during embryogenesis and cancer progression. Cancer Sci 98: 1512-1520, 2007.

6. Shinto O, Yashiro M, Kawajiri H, et al: Inhibitory effect of a TGFbeta receptor type-I inhibitor, Ki26894, on invasiveness of scirrhous gastric cancer cells. Br J Cancer 102: 844-851, 2010.

7. Kalluri R and Weinberg RA: The basics of epithelial-mesenchymal transition. J Clin Invest 119: 1420-1428, 2009.

8. Gordaliza M: Natural products as leads to anticancer drugs. Clin Transl Oncol 9: 767-776, 2007.

9. Boose G and Stopper H: Genotoxicity of several clinically used topoisomerase II inhibitors. Toxicol Lett 116: 7-16, 2000.

10. Newman D, Cragg G and Snader K: The influence of natural products upon drug discovery. Nat Prod Rep 17: 215-234, 2000.

11. Carmady B and Smith CA: Use of Chinese medicine by cancer patients: a review of surveys. Chin Med 9: 22, 2011.

12. Chinese Pharmacopoeia Commission (ed.). Pharmacopoeia of the People's Republic of China. Vol. 1. Chinese Medical Science and Technology Press, Beijing, pp573-575, 2010.

13. Xu YY and Yu EX: Clinical analysis of the effect of Pien Tze Huang in treatment of 42 patients with moderate or advanced liver cancer. Shanghai J Tradit Chin Med 12: 4-5, 1994.

14. Gu ZX: Therapeutical observation of advanced colon cancer. Chin Tradit Patent Med 15: 23, 1993.

15. Lin JM, Wei LH, Chen YQ, Liu XX, Hong ZF, Sferra TJ and Peng J: Pien Tze Huang induced apoptosis in human colon cancer HT-29 cells is associated with regulation of the Bcl-2 family and activation of caspase 3. Chin J Integr Med 17: 685-690, 2011.

16. Zhuang QC, Hong F, Shen AL, et al: Pien Tze Huang inhibits tumor cell proliferation and promotes apoptosis via suppressing the STAT3 pathway in colorectal cancer mouse model. Int J Oncol 40: 1569-1574, 2012.

17. Shen AL, Hong F, Liu LY, Lin JM, Zhuang QC, Hong ZF and Peng J: Effects of Pien Tze Huang on angiogenesis in vivo and in vitro. Chin J Integr Med 18: 431-436, 2012. 
18. Zeisberg $\mathrm{M}$ and Neilson EG: Biomarkers for epithelial-mesenchymal transitions. J Clin Invest 119: 1429-1437, 2009.

19. Gumbiner BM: Regulation of cadherin-mediated adhesion in morphogenesis. Nat Rev Mol Cell Biol 6: 622-634, 2005.

20. Elliott RL and Blobe GC: Role of transforming growth factor Beta in human cancer. J Clin Oncol 23: 2078-2093, 2005.

21. Massagué J: TGFbeta signaling: receptors, transducers, and Mad proteins. Cell 85: 947-950, 1996.

22. de Caestecker MP, Piek E and Roberts AB: Role of transforming growth factor-beta signaling in cancer. J Natl Cancer Inst 92: 1388-1402, 2000.

23. Massagué J, Blain SW and Lo RS: TGFbeta signaling in growth control, cancer, and heritable disorders. Cell 103: 295-309, 2000

24. Wang J, Sergina N, Ko TC, Gong J and Brattain MG: Autocrine and exogenous transforming growth factor beta control cell cycle inhibition through pathways with different sensitivity. J Biol Chem 279: 40237-40244, 2004.

25. Oft M, Heider KH and Beug H: TGF-beta signaling is necessary for carcinoma cell invasiveness and metastasis. Curr Biol 8: 1243-1252, 1998.
26. Wang J, Sun L, Myeroff L, et al: Demonstration that mutation of the type II transforming growth factor beta receptor inactivates its tumor suppressor activity in replication error-positive colon carcinoma cells. J Biol Chem 270: 22044-22049, 1995.

27. Wang J, Han W, Zborowska E, et al: Reduced expression of transforming growth factor beta type I receptor contributes to the malignancy of human colon carcinoma cells. J Biol Chem 271: 17366-17371, 1996.

28. Fukushima T, Mashiko M, Takita K, et al: Mutational analysis of TGF-beta type II receptor, Smad2, Smad3, Smad4, Smad6 and Smad7 genes in colorectal cancer. J Exp Clin Cancer Res 22: 315-320, 2003.

29. Miyaki M, Iijima T, Konishi M, et al: Higher frequency of Smad4 gene mutation in human colorectal cancer with distant metastasis. Oncogene 18: 3098-3103, 1999.

30. van Roy F and Berx G: The cell-cell adhesion molecule E-cadherin. Cell Mol Life Sci 65: 3756-3788, 2008.

31. Miyoshi J and Takai Y: Structural and functional associations of apical junctions with cytoskeleton. Biochim Biophys Acta 1778: 670-691, 2008 